\title{
Pressure and Temperature Dependence of Threshold Current in Semiconductor Lasers Based on InGaAs/GaAs Quantum-Well Systems
}

\author{
M. Maziarz ${ }^{a, b}$, B. Piechal ${ }^{b}$, A. Bercha ${ }^{b}$, R. Bohdan $^{b}$, \\ W. TRzeCIAKOWSKI ${ }^{b}$ AND J.A. MAJEWSKI ${ }^{a}$ \\ ${ }^{a}$ Institute of Theoretical Physics, Faculty of Physics, Warsaw University \\ Hoża 69, 00-681 Warszawa, Poland \\ ${ }^{b}$ Institute of High Pressure Physics Unipress, Polish Academy of Sciences \\ Sokołowska 29, 01-142 Warszawa, Poland \\ In the present paper we demonstrate that wide-range wavelength tun- \\ ing of semiconductor lasers can be achieved by applying high pressure and \\ low temperature. We report the experimentally measured dependence of \\ the threshold current and emission energy on pressure and temperature in \\ InGaAs/GaAs quantum-well lasers and provide the simple theoretical expla- \\ nation of the physics behind the experimental findings.
}

PACS numbers: 42.55.Px, 85.60.Jb

\section{Introduction}

Wavelength tuning of laser diodes is important in several areas of modern technology, such as telecommunication (e.g., dense wavelength division multiplexing), gas detection (e.g., tunable diode laser absorption spectroscopy), and medicine (e.g., photo-dynamic therapy). Diode lasers can be tuned by external or internal grating, just forcing the laser to oscillate at the frequency determined by the grating [1]. This method allows for tuning within the gain curve. The second possibility is to shift the whole gain curve of the laser. This can be achieved by the application of high pressure or by the modulation (typically lowering) of the temperature. The tuning range is limited by the variation of threshold current. For example, increased temperature is not practical for diode laser tuning because the threshold current increases rapidly and the lifetime decreases. The pressure dependence of threshold current is governed by the recombination mechanisms in the device. In the present paper, we analyze experimentally and theoretically the pressure and temperature dependence of radiative recombination in a typical 
$980 \mathrm{~nm}$ InGaAs/GaAs laser diode. For the wavelength range of 850-1100 nm, it can be assumed that the Auger recombination is negligible. Also the leakage currents are negligible because of a large separation between the Fermi level in the InGaAs quantum well (QW) and the barriers in GaAs waveguides and AlGaAs claddings. Therefore the radiative recombination in the quantum well should constitute the major component of the threshold current in $980 \mathrm{~nm}$ devices. The experimental data have been obtained in the temperature range of 100-300 $\mathrm{K}$ and in the pressure range of 0-20 kbar [2]. They are compared with the predictions of a simple model that not only sheds light on the physical mechanisms of the pressure and temperature tuning of lasers but also gives fairly reasonable quantitative results.

\section{Experimental details}

The investigated laser diodes (LDs) were single-mode ridge devices with a length of $300 \mu \mathrm{m}$ and a width of the ridge of $6.5 \mu \mathrm{m}$. They consisted of the active layer containing $\mathrm{In}_{0.2} \mathrm{Ga}_{0.8} \mathrm{As}$ QW of a width $8 \mathrm{~nm}$ (compressively strained), the $300 \mathrm{~nm}$ wide $\mathrm{GaAs}$ waveguide, and the claddings made of $\mathrm{Al}_{0.3} \mathrm{Ga}_{0.7} \mathrm{As}$ doped with $\mathrm{Si}$ and $\mathrm{Zn}$, for $n$-type and $p$-type cladding, respectively. The chips were mounted $p$-side down with $\mathrm{In}$ on $\mathrm{Cu}$ heat sinks. The heat sinks were placed on the plug to the high-pressure cell and we used the fiber feed through to pass the light out of the cell. The cell was filled with gasoline and closed with the piston. The cooling system was based on a spiral made of copper tubes surrounding the pressure cell. Liquid nitrogen was passed through the tubes to cool down the cell and the wire heater was used to stabilize the temperature. Thus, at a given pressure, the cell was placed in the cooling system and the spectra and $L-I-V$ characteristics were measured at different temperatures. Usually, the pressure dropped while cooling the cell, but this effect was taken into account evaluating the experimental data. Then the cell was heated up, the pressure was increased (at room temperature) and the next cooling cycle was performed. The spectra were measured on a SPEX1000M monochromator with a CCD.

\section{Simple model}

The simple qualitative understanding of the pressure and temperature dependence of the threshold current in QW LDs is based on the analytical expressions for the band edge peak gain, $G_{\max }$, for electron and hole density $n$ at the QW band edge [3]:

$$
G_{\max }=G_{0}\left[f_{\mathrm{c}}(n)-f_{\mathrm{v}}(n)\right],
$$

where $f_{\mathrm{c}}$ and $f_{\mathrm{v}}$ are the Fermi-Dirac distribution functions for electrons at the conduction and valence band edges, respectively, and $G_{0}$ is given by

$$
G_{0}=\frac{\Gamma E_{\mathrm{g}} \mu^{2} m_{\mathrm{r}}}{\varepsilon_{0} c n_{1} \hbar^{3} L_{z}},
$$

where the spatial overlap integral has been dropped out in $G_{0}, E_{\mathrm{g}}$ is the energy 
gap, $\mu$ is dipole moment matrix element for the transition between the conduction and valence bands edges, $m_{\mathrm{r}}$ is the reduced mass, $L_{z}$ is the well width, $\varepsilon_{0}$ is the dielectric constant of the vacuum, $n_{1}$ is the refractive index of the material, and $\Gamma$ is the optical confinement factor.

The band edge peak gain $G_{\max }$ can be rewritten using the inverted forms of the Fermi occupation factors as

$$
G_{\max }=G_{0}\left(1-\mathrm{e}^{-n / n_{\mathrm{c}}}-\mathrm{e}^{-n / n_{\mathrm{v}}}\right) \quad \text { with } \quad n_{\mathrm{c}, \mathrm{v}}=\frac{m_{\mathrm{c}, \mathrm{v}}}{\pi \hbar^{2} L_{z}} k_{\mathrm{B}} T
$$

where $m_{\mathrm{c}, \mathrm{v}}$ is the conduction or valence band effective mass, respectively, and the relation between the electron density $n$ and the Fermi occupation factor $f_{\mathrm{c}}$ is given below (for hole density $\mathrm{c} \rightarrow \mathrm{v}$ ):

$$
n=\sum_{l} n_{\mathrm{c}} \log \left(1+\frac{f_{\mathrm{c}}}{1-f_{\mathrm{c}}} \mathrm{e}^{-E_{\mathrm{c} l}}\right) .
$$

We assume that all subbands $l$ are parabolic and that optical transitions obey $k$-selection rules. Following the $k \cdot p$ theory, we assume that the electron and light hole effective masses are proportional to the band gap $E_{\mathrm{g}}$, while effective mass of heavy holes is assumed constant. Thus, since the energy gap $E_{\mathrm{g}}$ increases with hydrostatic pressure, the effective masses of electrons and light holes follow this trend. Also the temperature dependence of the band gaps is the base for establishing the temperature dependence of other quantities. The data from Refs. $[4-6]$ have been used through this paper to determine the temperature and pressure dependence of quantities defined in Eqs. (1)-(4). Specifically, we have used the temperature and pressure independent refractive index equal to $n_{1}=3.57$, the parameter $E_{\mathrm{p}}=23 \mathrm{eV}$, and the heavy hole effective mass of $0.36 m_{0}$. The pressure and temperature dependence of the energy gap $E_{\text {gap }}$ (in $\mathrm{eV}$ ), the conduction band effective mass $m_{\mathrm{e}}$ (in $m_{0}$ ), and the optical confinement factor $\Gamma$ are given by the following formulae:

$$
\begin{aligned}
& E_{\text {gap }}=1.232+0.0104 p-\frac{0.00068 T^{2}}{480+T}, \\
& \frac{1}{m_{\mathrm{e}}}=\frac{E_{\mathrm{p}}}{3}\left(\frac{2}{E_{\text {gap }}}+\frac{1}{E_{\text {gap }}+\Delta_{0}}\right), \\
& \Gamma=0.02084+0.00034 p+2 \times 10^{-6} T,
\end{aligned}
$$

where the hydrostatic pressure $p$ is expressed in kbar, the temperature $T$ in $\mathrm{K}$, and the valence band spin splitting $\Delta_{0}$ is equal to $0.345 \mathrm{eV}$. Let us note that the dipole moment $\mu$ is related to the parameter $E_{\mathrm{p}}$ through the relation $\mu^{2}=$ $E_{\mathrm{p}} e^{2} \hbar^{4} / 2 m_{0}^{3} E_{\mathrm{g}}^{2}$.

The simple scheme described above allows the calculation of the maximum gain as a function of carrier density in the well [3, 7], and thus its dependence on the temperature and hydrostatic pressure. Assuming that the dominant carrier recombination process is the radiative recombination (i.e., neglecting nonradiative 


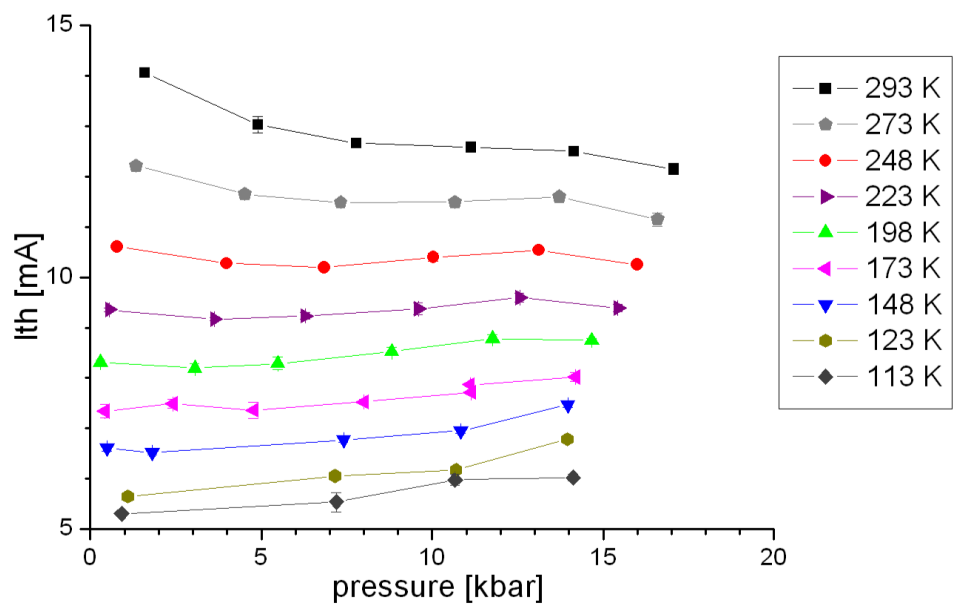

Fig. 1. Experimental threshold current vs. pressure at various temperatures.

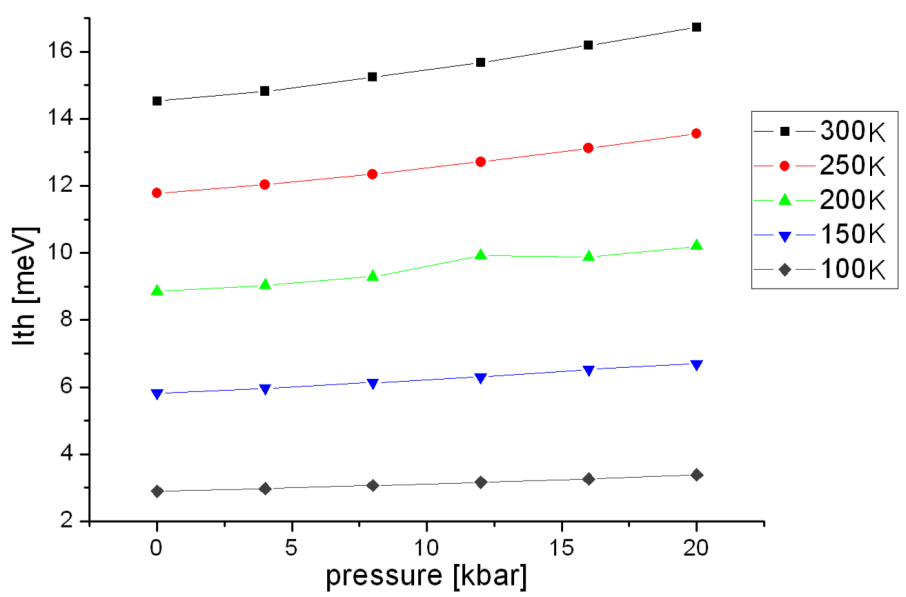

Fig. 2. Theoretical threshold current vs. pressure at various temperatures.

recombination paths such as the Auger recombination), the radiative part of the threshold current density can be written as $[3,7]$

$$
j_{\mathrm{th}}^{\mathrm{rad}}=e L_{z} B n_{\mathrm{th}}^{2},
$$

where $B$ is the bimolecular radiative recombination coefficient between electrons and holes

$$
B=\frac{2 \pi e^{2} n_{1} E_{\mathrm{p}} E_{\mathrm{g}} L_{z}}{3 m_{0}^{2} c^{3} k_{\mathrm{B}} T} \frac{1}{m_{\mathrm{hh}}+m_{\mathrm{lh}}}\left(\frac{m_{\mathrm{hh}}}{m_{\mathrm{hh}}+m_{\mathrm{c}}}+\frac{m_{\mathrm{lh}}}{m_{\mathrm{lh}}+m_{\mathrm{c}}}\right),
$$

and $n_{\mathrm{th}}$ is the threshold carrier density. Assuming that the $G_{\max }$ is equal to the losses (optical and on mirrors), $n_{\text {th }}$ has been determined from Eq. (3). The estimated losses have been taken to be equal to $17.04 \mathrm{~cm}^{-1}$, and possible pressure 


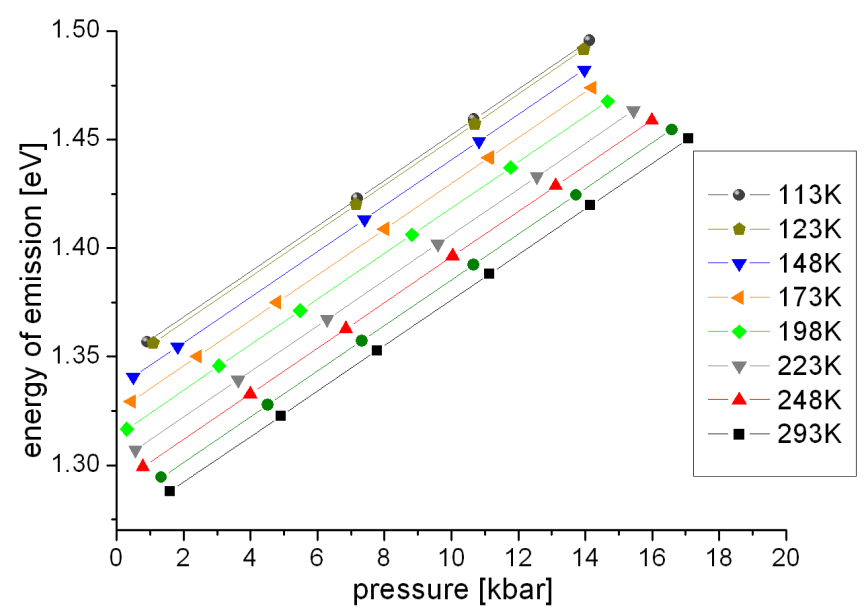

Fig. 3. Experimental emission energy vs. pressure at various temperatures.

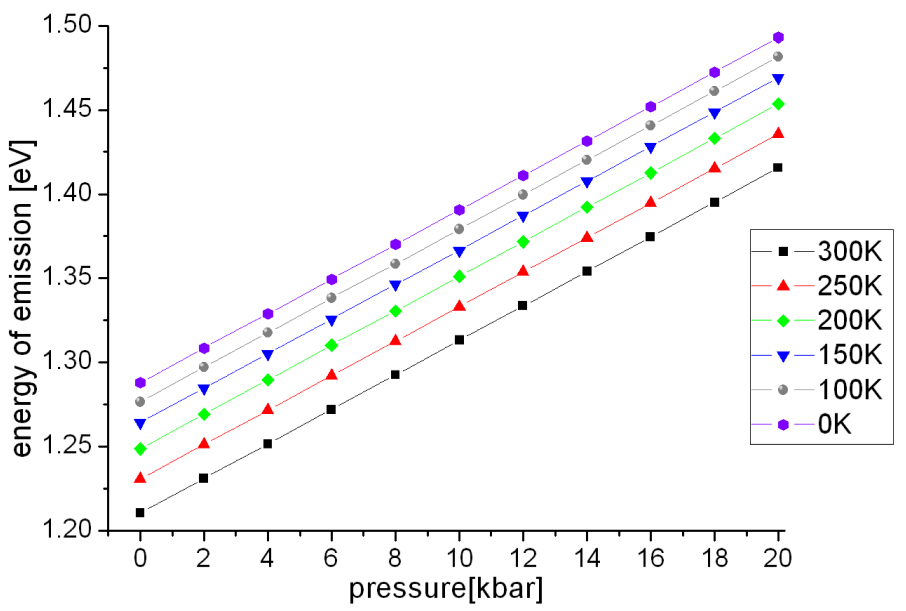

Fig. 4. Theoretical emission energy vs. pressure at various temperatures.

and temperature dependences of the losses have been neglected for these considerations. Let us note that the transparency occurs when $G_{\max }=0$. Thus the transparency carrier density $n_{\mathrm{tr}}$, and its dependence on the temperature and hydrostatic pressure, can be determined from Eqs. (1)-(4) in analogous way to $n_{\mathrm{th}}$.

In Fig. 1, we show the experimental results of the threshold current as a function of hydrostatic pressure at a few temperatures, whereas in Fig. 2 the calculated variations of the threshold current with pressure and temperature are depicted. As one can see, the threshold current increases with pressure and temperature and the agreement between theory and experiment is rather good.

In Figs. 3 and 4, the dependence of the energy of emission on pressure and temperature is depicted, as extracted from experiments and calculated theoreti- 
cally. Theoretical emission energies have been obtained by subtracting the highest subband energy of the holes from the lowest subband energy of electrons, both obtained from quantum mechanical calculations for the QW. In experiments, the energy of emission has changed about $200 \mathrm{meV}$ with hydrostatic pressure, which means that the laser light wavelength could be tuned in the range from $980 \mathrm{~nm}$ to $840 \mathrm{~nm}$. Theoretical calculations give an increase in emission energy by about $205 \mathrm{meV}$, which agrees excellently with experimental data. The tuning range of the emission energy with temperature (from $300 \mathrm{~K}$ to $100 \mathrm{~K}$ ) obtained in experiment and theory was in excellent agreement, and was equal to 80 and $70 \mathrm{meV}$, respectively.

\section{Conclusions}

The experimental and theoretical studies of lasers based on InGaAs/GaAs heterostructures demonstrate that the possible tuning range of the emission energy is about $200 \mathrm{meV}$, if one uses $20 \mathrm{kbar}$ cell, and $80 \mathrm{meV}$, if the device is cooled down from room temperature to $100 \mathrm{~K}$. The increase in the emission energy happens also with almost constant output power in the whole tuning range. The studies clearly show that the presented simple model describes very well not only qualitative physics but also gives quantitative predictions that are in fairly good agreement with experiments.

\section{Acknowledgments}

This work was supported by the European Commission through the Integrated Project WWW.BRIGHTER.EU (grant referenced FP6-IST-035266).

\section{References}

[1] M.-C. Amann, J. Buus, Tunable Laser Diodes, Artech House, Boston 1998.

[2] W. Trzeciakowski, A. Bercha, F. Dybała, R. Bohdan, P. Adamiec, O. Mariani, Phys. Status Solidi B 244, 179 (2007).

[3] K.J. Vahala, C.E. Zah, Appl. Phys. Lett. 52, 1945 (1988).

[4] O. Madelung, Semiconductor Basic Data, Springer Verlag, Berlin 1996.

[5] S. Adachi, Optical Constants of Crystalline and Amorphous Semiconductors, Kluwer Academic Publishers, Boston 1999.

[6] www.ioffe.rssi.ru.

[7] B. Gönül, M. Oduncuoğlu, Semicond. Sci. Technol. 19, 23 (2004). 